\title{
Data-Driven Flow-Map Models for Data-Efficient Discovery of Dynamics and Fast Uncertainty Quantification of Biological and Biochemical Systems
}

\author{
Georgios Makrygiorgos $^{1,2}$ | Aaron J. Berliner ${ }^{1,3}$ | \\ Fengzhe Shi $^{1,2}$ | Douglas S. Clark ${ }^{1,2}$ | Adam P. Arkin ${ }^{1,3}$ \\ | Ali Mesbah ${ }^{1,2}$
}

\author{
${ }^{1}$ Center for the Utilization of Biological \\ Engineering in Space (CUBES), \\ http://cubes.space/ \\ ${ }^{2}$ Department of Chemical and Biomolecular \\ Engineering, University of California, \\ Berkeley, CA 94720, USA \\ ${ }^{3}$ Department of Bioengineering, University \\ of California, Berkeley, CA 94720, USA
}

\section{Correspondence}

Ali Mesbah

Email: mesbah@berkeley.edu

\section{Funding information}

This material is based upon work supported by NASA under grant or cooperative agreement award number NNX17AJ31G.
Computational models are increasingly used to investigate and predict the complex dynamics of biological and biochemical systems. Nevertheless, governing equations of a biochemical system may not be (fully) known, which would necessitate learning the system dynamics directly from, often limited and noisy, observed data. On the other hand, when expensive models are available, systematic and efficient quantification of the effects of model uncertainties on quantities of interest can be an arduous task. This paper leverages the notion of flow-map (de)compositions to present a framework that can address both of these challenges via learning data-driven models useful for capturing the dynamical behavior of biochemical systems. Data-driven flow-map models seek to directly learn the integration operators of the governing differential equations in a black-box manner, irrespective of structure of the underlying equations. As such, they can serve as a flexible approach for deriving fast-toevaluate surrogates for expensive computational models of system dynamics, or, alternatively, for reconstructing the long-term system dynamics via experimental observations. We present a data-efficient approach to data-driven flow-map modeling based on polynomial chaos Kriging. The approach is demonstrated for discovery of the dynamics of various benchmark systems and a co-culture bioreactor subject to external forcing, as well as for uncertainty quantification of a microbial electrosynthesis reactor. Such data-driven models and analyses of dynamical systems can be paramount in the design and optimization of bioprocesses and integrated biomanufacturing systems.

\section{KEYWORDS}

Flow-map decomposition; Probabilistic surrogate modeling; Discovery of nonlinear dynamics; Uncertainty quantification; Polynomial chaos Kriging 


\section{1 | INTRODUCTION}

Computational models have become indispensable tools for understanding the complex behavior of biological and biochemical systems towards design and optimization of bioprocesses and integrated biomanufacturing systems [1]. Recently, there has been a growing interest in data-driven methods for modeling the uncertain and nonlinear dynamics of biochemical systems, as these models constitute the cornerstone of various model-based analyses and decision-making tasks such as experiment design, hypothesis testing and parameter inference [2, 3, 4]. Data-driven modeling is especially useful when it is formidable to derive firstprinciples descriptions for systems whose complex behavior can span over multiple length- and time-scales. Data-driven models have shown promise for inferring the dynamics of cellular systems and metabolic networks (e.g., [5, 6]). Hybrid models (aka gray-box models) that combine physics-based models with data-driven descriptions of unknown or hard-to-model phenomena have also proven useful for describing the complex behavior of biochemical systems $[7,8,9,10]$.

In this work, we focus on data-driven discovery of dynamical systems, whereby the goal is to learn directly the governing equations from system observations. A class of data-driven discovery methods for unknown systems relies on basic assumptions about the structure of the underlying equations [11]. To this end, a popular technique is based on sparse identification from dictionaries of possible governing terms [12, 13], which has been shown to be particularly useful when limited system observations are available. On the other hand, nonparametric modeling approaches relax the necessity of using a library of candidate terms [14]. Another class of methods for data-driven reconstruction of dynamics is based on dynamic mode decomposition [15, 16], which approximates the eigenvalues and eigenvectors of the Koopman operator [17] that describes the dynamics of nonlinear systems.

Although inception of the field of nonlinear system identification dates back to few decades ago [18], the advent of machine learning, in particular deep learn- ing, for characterizing complex input-output relationships has reinvigorated the interest in this area. Most notably, physics-informed neural networks [19] and dynamics reconstruction via neural networks under noisy data [20] have shown promise for data-driven modeling of nonlinear dynamical systems. Recently, Qin et al. [21, 22] proposed a deep learning-based approach for data-driven approximation of integration operator of differential equations from observations of state variables. The usefulness of this approach for discovery of dynamics of biological systems has been demonstrated on several benchmark problems in [23], mainly since it removes the necessity of assumptions about the dynamic model structure.

Data-driven discovery methods can also be used for model-based uncertainty quantification (UQ) applications that rely on expensive-to-evaluate computational models. Predictions of the behavior of biochemical systems are generally subject to various sources of uncertainty due to unknown model structure, parameters, and/or initial and boundary conditions. Systematic and accurate quantification of the effects of these uncertainties on predictions of quantities of interest (Qols) is crucial when using models for decision-support tasks. This has spurred development of a plethora of set-based [24] and probabilistic $[25,26]$ methods for forward and inverse UQ problems (e.g., [27, 28, 29, 30, 31]). However, the most commonly used UQ methods rely on Monte Carlo sampling [32], which can be intractable for expensive computational models of biochemical systems, especially when models consist of a large number of differential equations and/or have a large number of uncertain inputs.

Surrogate modeling is being increasingly used to facilitate complex UQ analyses that would otherwise be computationally prohibitive. The key notion in surrogate modeling is to construct a data-driven mapping between inputs to a system and the Qols in a non-intrusive manner, in which the "data generating process," e.g., a high-fidelity model, is treated as a black-box to generate as few training samples as possible [33]. Such a data-driven representation can be used as a computationally efficient surrogate for expensive computational 
models in order to predict the Qols as a function of inputs. A variety of surrogate modeling techniques such a generalized and sparse polynomial chaos [34, 35], Kriging [36] and deep learning [37] have been successfully applied to various biological and biochemical systems (e.g., [31, 38, 39, 40, 41]). Nonetheless, a critical challenge in the majority of these techniques arises from capturing the time-evolution of the Qols in an efficient manner. The most common approach, known as timefrozen surrogate modeling [42, 43], for predicting the time-evolution of Qols relies on constructing separate surrogate models for all time points at which the Qols must be predicted. As such, the "time-frozen" approach can be an inflexible and inefficient way of surrogate modeling for dynamical systems, especially in dynamic UQ and decision-making problems that hinge on making predictions over an adaptive sequence of time instants.

In this paper, we leverage the notion of flow-map (de)composition, as also investigated in [21, 22], for data-efficient discovery of system dynamics from experimental observations or high-fidelity simulation data. Conceptually, a flow-map is an analytical operator that maps the current state and input of a system to a future state based on exact integration of model equations over some specified time step. Numerical integration schemes for ordinary differential equations in fact seek to numerically approximate flow-maps to compute the time-evolution of state variables as a function of input variables. Here, we propose to approximate flowmaps in a data-driven manner via non-intrusive surrogate modeling, such that the resulting data-driven flowmap is a surrogate for differential operators of the differential equations governing a dynamical system. Hence, data-driven flow-map models are able to discover system dynamics irrespective of the unknown structure of model equations. In addition, data-driven flow-map models can address the above-described challenge of "time-frozen" approaches to surrogate modeling via circumventing the need for construction of separate surrogate models at different time instants. This can be especially useful for fast UQ and optimization-based analyses of dynamical systems that hinge on repeated runs of expensive computational models over a sequence of time instants.

We demonstrate the usefulness of data-driven flowmaps for discovery of system dynamics from data, as well as for fast UQ applications based on expensive computational models. In this work, sparse polynomial chaos Kriging [44] is used for data-driven approximation of flow-maps owing to its data efficiency, ability to approximate complex mappings and ability to quantify the uncertainty of model predictions. The versatility of datadriven flow-maps is first demonstrated via the discovery of the transient behavior of benchmark problems and a co-culture bioreactor using noisy data. Subsequently, we show how data-driven flow-maps can speedup forward and inverse UQ analyses of a dynamic microbial electrosynthesis reactor, achieving up to a 100 -fold gain in computational speed.

\section{2 | METHODS}

In this section, we present the idea of flow-map (de)composition for dynamical nonlinear systems. This is followed by a discussion on the surrogate modeling technique and data generation strategies used in this work for learning data-driven flow-map models.

\subsection{Flow-map Compositions}

Consider a dynamical, time-invariant, nonlinear system described by

$$
\frac{d s}{d t}=f(s, x), \quad s(t=0)=s_{0},
$$

where $s \in \mathbb{R}^{n_{s}}$ is the vector of state variables with initial conditions $s_{0}, x \in \mathbb{R}^{n_{x}}$ is the vector of input variables, and $\boldsymbol{f}(\boldsymbol{s}, \boldsymbol{x}): \mathbb{R}^{n_{s}} \times \mathbb{R}^{n_{x}} \rightarrow \mathbb{R}^{n_{s}}$ is the vector of (possibly unknown) system equations; $\mathbb{R}$ denotes the set of real numbers. Eq. (1) describes the time-evolution of the state, $s$, of the nonlinear system as a function of the inputs $\boldsymbol{x}$. Notice that in this work the inputs $\boldsymbol{x}$ can represent either model parameters, or manipulated input variables to a biochemical system, as will be discussed later. 
A flow-map function is a mapping that predicts the transition of a dynamical system from the current to future state [21]. We define a flow-map function $\Phi_{\delta}$ as

$$
s(\delta ; x)=\Phi_{\delta}\left(s_{t}, x\right)
$$

where $s_{t}$ denotes the current state at time $t$ and $\delta$ is the lag time in the system transition from the current state $s_{t}$ to the future state $s$. Given the current state of a system at time $t, \Phi_{\delta}$ is in fact an analytical operation based on exact integration of $\boldsymbol{f}$, yielding the state after time $\delta$

$$
\boldsymbol{s}(\delta ; \boldsymbol{x})=\boldsymbol{s}(t ; \boldsymbol{x})+\int_{t}^{t+\delta} \boldsymbol{f}\left(\boldsymbol{s}\left(t^{\prime} ; \boldsymbol{x}\right), \boldsymbol{x}\right) d t^{\prime}
$$

Eq. (3) describes the one-step transition between the states of a system. The integral term that appears in (3) can, subsequently, be considered as a flow-map residual, i.e., it represents the discrepancy between the current and future set of states.

The notion of flow-map compositions can be applied to compose a sequence of one-step transitions to define state trajectories over time [21]. Once the $\delta$-lag flowmap $\Phi_{\delta}$ is established, it can be used to predict states $s$ at any time instant $\Delta=\sum_{j=1}^{K} \delta_{j}$ using a K-fold composition

$$
\Phi_{\Delta}=\Phi_{\delta_{K}} \circ \cdots \circ \Phi_{\delta_{1}}
$$

In practice, the set of differential equations in Eq. (1) 185 describing the system dynamics may not be known, or, $\quad{ }_{186}$ when known, their numerical solution may be expensive. $\quad \mathbf{1 8 7}$ In this paper, we aim to learn an approximate surrogate $\quad \mathbf{1 8 8}$ for the flow-map function in Eq. (2) from high-fidelity 189 simulation or experimental data. Data-driven flow-map $\quad 190$ models can be established from simulation data to pro- $\quad 191$ vide an efficient surrogate for expensive computational 192 models of the form in Eq. (1) that, for example, rely on 193 numerical integration of a large number of highly nonlin- 194 ear and stiff differential equations, as is commonly the $\mathbf{1 9 5}$ case for complex biochemical systems. Notice that in 196 this case data-driven flow-map modeling can be viewed as approximating numerical time integrators of the differential equations in Eq. (1). Alternatively, in the absence of any knowledge about the governing equations (i.e., functions $\boldsymbol{f}$ in Eq. (1)), flow-map models can be directly learned from experimental observations in order to discover the unknown system dynamics. The main steps of data-driven flow-map modeling are summarized as follows. First, observations of the state variables are collected at several time instants either using highly-fidelity simulations, or via performing experiments. Notice that there is usually some degree of freedom in choosing the lag time $\delta$ in simulations, whereas the choice of $\delta$ is often limited by how fast measurements can be acquired in experiments. Then, the observations of the state trajectories over a sequence of discrete-time instants are used to train a surrogate for

88

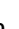
91

\section{2}

\section{3} 94 95

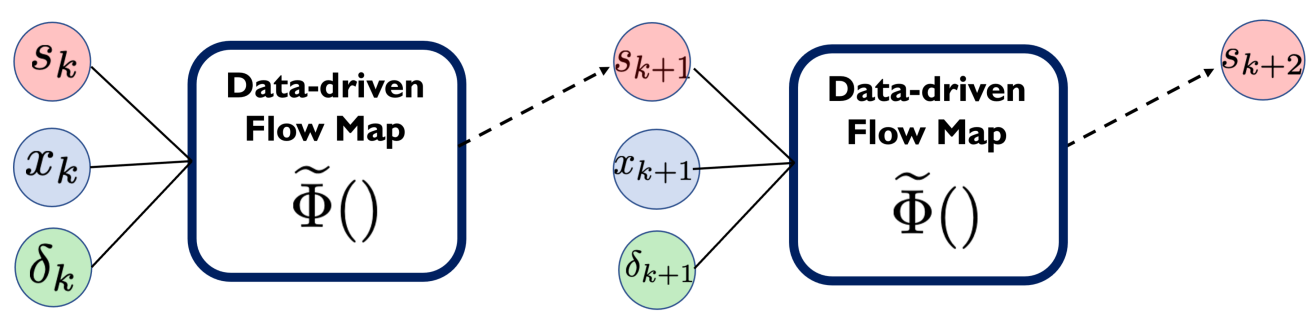

F I G URE 1 Data-driven flow-map models for predicting the state variables of a dynamical system over time. The flow-map model $\widetilde{\Phi}$ takes the current states $\boldsymbol{s}_{k}$, inputs $\boldsymbol{x}_{k}$, and lag time $\delta_{k}$ at a discrete-time instant $k$ as inputs to predict the states $\boldsymbol{s}_{k+1}$ at the subsequent time instant $k+1$. By sequentially repeating this procedure, the time-evolution of the states in relation to the inputs can be established. 


$$
\boldsymbol{y}=\widetilde{\Phi}(\boldsymbol{w})=\sum_{\boldsymbol{a} \in \mathbb{N} M} y_{a} P_{\boldsymbol{a}}(\boldsymbol{W})+\sigma^{2} Z(\boldsymbol{w}),
$$

where $\boldsymbol{Y} \in \mathbb{R}^{n_{s}}$ denotes the Qols at $k+1$ that are typically a subset of the states $s ; P_{a}(\boldsymbol{W})$ are multivariate polynomial basis functions that are orthogonal with re- spect to the probability distribution $f_{W}$ over the support $\mathcal{D}_{W}$ of the distribution, i.e.,

$$
\begin{array}{r}
\mathbb{E}\left\{P_{i}(\boldsymbol{W}) P_{j}(\boldsymbol{W})\right\} \\
=\int_{\mathcal{D}_{W}} P_{i}(\boldsymbol{w}) P_{j}(\boldsymbol{w}) f_{W}(\boldsymbol{w}) d \boldsymbol{w}=\delta_{i j}, \\
\forall i, j \in \mathbb{N}^{M},
\end{array}
$$

with $\mathbb{E}$ being the expectation operator and $\delta_{i j}$ the Kronecker delta; $y_{a}$ are the coefficients of the basis functions, with the multi-index $a$ being an $M$-dimensional vector in the set of natural numbers $\mathbb{N} ; Z(\boldsymbol{w})$ is a standard normal random; and $\sigma^{2}$ is a variance hyperparameter of the PCK.

The PCK in Eq. (5) represents the Qols $\boldsymbol{y}$ as a Gaussian process (GP), such that the first term in Eq. (5) describes the trend (or mean) of the GP while the second term $Z(\boldsymbol{w})$ describes the variance of the predicted Qols. The trend of PCK is in fact an expansion of orthogonal polynomials that can represent any finite variance Qol [50]. Constructing the orthogonal basis $P_{a}(W)$ requires the knowledge of the multivariate probability distribution $f_{W}$. Eq. (6) gives the tensor product of $M$ univariate polynomials that are orthonormal with respect to their corresponding marginal probability distribution. Optimal $L_{2}$-convergence of the expansion of orthogonal polynomials has been established based on the Wiener-Askey scheme for various probability distributions [50, 51], although arbitrary orthogonal basis functions with sub-optimal convergence can also be constructed directly from moments of the random variable $\boldsymbol{W}$ [52]. As described, the multivariate random variable $\boldsymbol{W}$ consists of the states $\boldsymbol{s}$, input variables $\boldsymbol{x}$, and time lag $\delta$. When $\boldsymbol{x}$ corresponds to uncertainties of a computational model (e.g., uncertainties in model parameters and/or initial conditions), their probability distribution is typically available $a$ priori from parameter inference. As such, their respective polynomial basis functions can be chosen according to the Wiener-Askey scheme (e.g., Hermite basis for Gaussian distributions, Legendre for uniform distributions). On the other hand, when $\boldsymbol{x}$ corresponds to manipulated variables of a system, as is the case in the discovery of system dynamics, the input vari- 
ables can typically be modeled as uniform distributions within a known range. The time lag $\delta$ can also be modeled as a uniform distribution within some range of interest for the application at hand. However, the distribution of states $s_{k}$ is dependent on the realized state trajectories when the training data are generated and, thus, cannot be established a priori. Here, we assume states follow a multivariate Gaussian distribution with a mean and covariance computed from the training samples.

For practical reasons, the expansion of the trend term in Eq. (5) must be truncated up to a finite order. The truncated polynomial chaos expansion takes the form

$$
\sum_{\boldsymbol{a} \in \mathcal{A}} y_{a} P_{a}(\boldsymbol{W})
$$

where the order of the expansion is dictated by the multi-index $a \in \mathcal{A}$, with $\mathcal{A} \subset \mathbb{N}^{M}$ being the set of the multi-indices kept in the truncated expansion. The truncation scheme aims to limit the infinite expansion of the trend to a series of maximum order $p$. To address the challenges that arise due to increasing the order of the polynomial basis for better approximation and/or the large dimension of $\boldsymbol{w}$, sparsity can be introduced by employing the hyperbolic truncation scheme [35], also known as the q-norm scheme,

$$
\begin{aligned}
& \mathcal{A}^{M, p, q}=\left\{\boldsymbol{a} \in \mathcal{A}^{M, p}:\|\boldsymbol{a}\|_{q} \leq p\right\}, \\
&\|\boldsymbol{a}\|_{q}=\left(\sum_{i=1}^{M} a_{i}^{q}\right)^{\frac{1}{q}} .
\end{aligned}
$$

In principle, the coefficients $y_{a}$ of the polynomial chaos expansion in Eq. (7) can be determined in a nonintrusive manner via solving a least-squares problem [53]. Here, we induce further sparsity by modifying the coefficient estimation problem to a $L_{1}$-regularized regression problem [54]. The regularized coefficient estimation problem can be efficiently solved using the leastangle-regression (LAR) algorithm [55], which efficiently estimates the coefficients of the most relevant terms of the expansion in Eq. (7), setting the rest of the coefficients to zero.
Moreover, $Z(\boldsymbol{w})$ in Eq. (5) is defined in terms of a 308 kernel function $R\left(\left|\boldsymbol{w}-\boldsymbol{w}^{\prime}\right|, \theta\right)$, i.e., a function that pro- 309 vides some measure of similarity between different re- $\quad 310$ alizations of the random variable $\boldsymbol{W}$. Here, we use the 311 Matérn kernel function [49]. Overall, the "tuning param- 312 eters" of the PCK that must be determined using the 313 training data include the coefficients $y_{a}$ of the trend, the variance term $\sigma^{2}$, and the hyperparameters $\theta$ of the kernel function. This is efficiently done via maximumlikelihood estimation [44].

Finally, to quantify the quality of the PCK predictions, we use the leave-one-out cross-validation (LOOCV) error that is estimated from the training data. When onestep ahead test samples are available, validation errors can readily be evaluated. Furthermore, we assess the ability of the data-driven flow-map models in approximating the integration operator and, hence, their predictive accuracy over a multi-step integration horizon. Given $i=1, \ldots, N_{V}$ validation state trajectories, each of which of length $T_{i}$, we define the normalized, timeaveraged prediction error of Qols, $\epsilon_{i}$, as

$$
\begin{aligned}
& \epsilon_{i}=\sum_{k=0}^{T_{i}} \frac{1}{T_{i}} \frac{\left\|\boldsymbol{y}_{k, i}-\boldsymbol{y}_{k, i}^{\text {true }}\right\|_{2}}{\left\|\boldsymbol{y}_{k, i}^{\text {true }}\right\|_{2}} \\
& \hat{\epsilon}=\frac{1}{N_{V}} \sum_{i=1}^{N_{v}} \epsilon_{i},
\end{aligned}
$$

where $\|\cdot\|_{2}$ is the 2-norm of a vector; $\boldsymbol{y}_{k, i}^{\text {true }}$ and $\boldsymbol{y}_{k, i}$ are, respectively, the vector of Ools in the validation dataset and those predicted by the data-driven flow-map models at time instant $k$ for each validation run $i$. In the remainder, we refer to $\epsilon_{i}$ as the mean trajectory error (MTE), whereas $\hat{\varepsilon}$ is the average MTE over all validation trajectories.

\section{3 | Data Generation and Model Training}

To train an approximate flow-map model $\widetilde{\Phi}\left(\boldsymbol{w}_{k}\right)$, we require input-output data that represent one-step transitions between states. To this end, a total of $N_{T}$ trajectories of state variables $s_{k}$ over a discrete-time horizon $\{0,1, \cdots, k, k+1, \cdots, T\}$ are generated, where $T$ is the

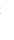

\section{9}

0


length of the time horizon of the training trajectories. At each time instant $k$, a single training sample consists of $\boldsymbol{w}_{k} \rightarrow \boldsymbol{y}_{k}$.

For trajectory generation, it is crucial to vary the initial conditions $\boldsymbol{s}_{0}$ and inputs $\boldsymbol{x}_{\boldsymbol{k}}$ within some allowable range, as well as the time lag $\delta$ whenever applicable. The training data must cover a wide range of state, input and time lag values, as relevant to the application of the trained models. As such, each sample of observed states within each trajectory represents a unique transition from the current to future state of the system for the given input and time lag values. We note that an effective strategy for generating simulation data is via

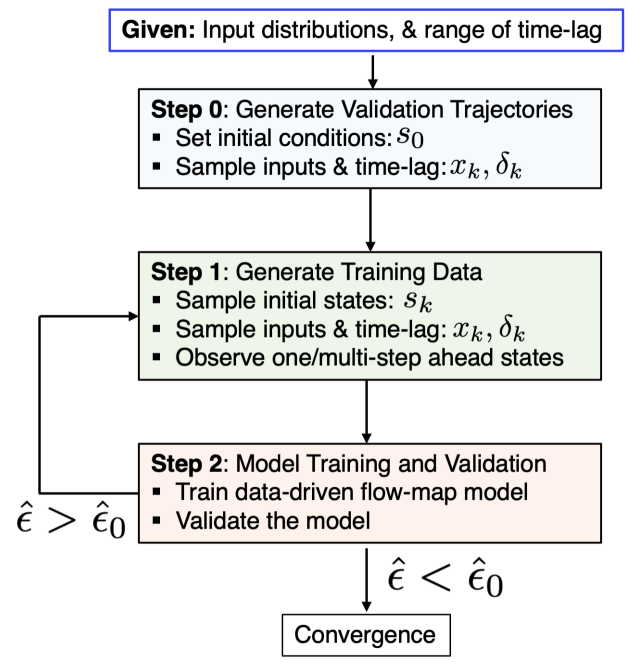

FIGURE 2 Algorithm for data generation and training of data-driven flow-map models. Validation trajectories are first generated. Then, one/multi-step ahead simulations or experiments are performed to observe successor states given the initial states, inputs, and time-lag. Subsequently, the data-driven flow-map model is trained. In the case of PCK models used in this work, several hyperparameters must be selected during the model training. These include the polynomial order, hyperbolic truncation parameter, covariance function and the regression method used for estimating the expansion coefficients. Finally, the prediction accuracy of the trained model is assessed against the long-time validation trajectories. If the prediction accuracy $\hat{\varepsilon}$ is larger than some pre-specified threshold $\hat{\epsilon}_{0}$, the model training and validation process will be repeated. one-step transitions. That is, instead of generating an entire trajectory given some initial conditions $\boldsymbol{s}_{0}$, we can randomly sample the state-space, along with the uncertain parameters and time lag, in order to compute the corresponding future states.

The data generation and PCK model training strategy adopted in this work is summarized in Figure 2. We remark that, although random sampling is used here to generate the training data, PCK provides confidence estimates on its predictions that can be used towards active learning-based sampling (e.g., see [56]). As will be demonstrated in the subsequent sections, the main benefits of using PCK for constructing data-driven flowmap models include: (i) being more data efficient, especially as compared to feedforward neural networks [23], when used for discovery of system dynamics from system observations; (ii) offering significant improvements in the computational efficiency of data generation for surrogate modeling for dynamical systems as compared to time-frozen approaches; and (iii) characterizing the uncertainty of model predictions.

In this work, the following procedure is used for fitting the PCK models. We use the sequential PC-Kriging approach proposed in [44], where a polynomial chaos expansion (PCE) is first trained based on the available data and is then embedded as the trend of PCK. For training the PCE, we allow the polynomial expansion's maximum order to vary from 1 to 5 ; higher order polynomials are avoided to retain a smaller expansion (i.e., less degrees of freedom) and mitigate overfitting. The truncation factor $q$ in Eq. (8) is varied from 0.7 to 0.85 since the resulting maximum order of the polynomials will ensure that we do not have highly nonlinear interaction terms while allowing for elimination of few of interaction terms. The optimal value of $q$ is chosen based on cross-validation. We use a Matérn kernel for the GP part of PCK models. The hyperparameters of PCK are selected using a data-driven optimization algorithm, namely the covariance matrix adaptation-evolution strategy [57]. 


\section{I DATA-DRIVEN DISCOVERY OF DYNAMICAL SYSTEMS}

In this section, we apply the PCK-based flow-map modeling approach to learn the dynamics of several benchmark systems using limited data. The first case study, based on the Morris-Lecar system, compares the performance of the PCK model with neural network modeling results of [23]. The second case study, based on the Lorenz system, focuses on reconstructing the dynamics of a chaotic system in which variations in parameters significantly change the solution landscape. Lastly, we show how the flow-map modeling approach can be used for discovering the dynamics of a co-culture bioreactor under noisy observations and how the variance term of PCK provides a measure of uncertainty of model predictions.

\section{1 | Morris-Lecar System}

The first benchmark problem is the Morris-Lecar system [58], which describes neuronal excitability. This system was used in [23] to examine neural network-based flowmap models for the discovery of nonlinear dynamics. In particular, a residual neural network was used to represent the data-driven flow-map model, in which only the flow-map residual is learned by skipping the input connection to the neural network and adding it to the output of the latter. Here, we aim to recreate the results of the aforementioned work, demonstrating the data efficiency of the proposed PCK approach to data-driven reconstruction of dynamics. The dynamics of the MorrisLecar system are described by

$$
\begin{aligned}
C_{M} \frac{d V}{d t}= & -g_{L}\left(V-V_{L}\right)-g_{C a}\left(V-V_{C_{a}}\right) M_{\infty} \\
& -g_{k}\left(V-V_{K}\right) N+I_{a p p} \\
\frac{d N}{d t}= & \lambda_{N}\left(N_{\infty}-N\right),
\end{aligned}
$$

where $V(\mathrm{mV})$ is the voltage difference between the sides of the membrane and $N$ represents the probability for the potassium channel being open. The parameters $M_{\infty}, N_{\infty}$ and $\lambda_{N}$ depend on the voltage, as defined

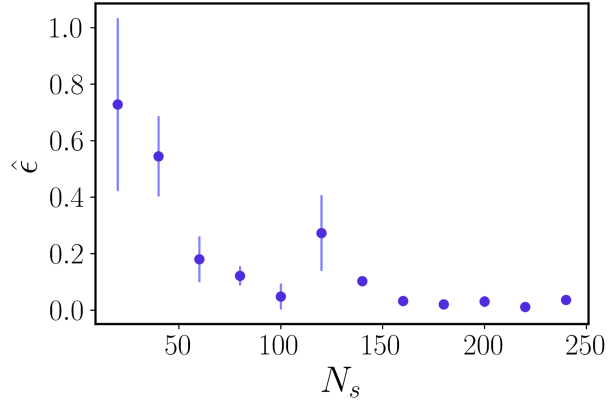

FIGURE 3 The average mean trajectory error, $\hat{\epsilon}$, of the PCK-based flow-map model for the Morris-Lecar system in relation to the number of training samples, $N_{s}$. The error is estimated based on three validation trajectories generated for the input $I_{a p p}$ values $\{0,60,150\}$. The vertical bars represent the standard deviation of the error estimated based on 5 repeats of the training.

in the SI. We focus on the so-called Type I model with parameters taken from [23] and given in the SI. Here, it is assumed that the model parameters are fixed, as we aim to reconstruct the system dynamics as a function of $x_{k}=I_{a p p}$ that can vary within the range $[0,300] \mathrm{A}$. Specifically, we aim to predict the long-term system dynamics, starting from a given initial conditions, under a fixed $I_{\text {app }}$. To compare our results with those in [23], $\delta_{k}$ was chosen to be $0.2 \mathrm{~ms}$; we did not consider the time-lag as part of the PCK model. This system exhibits a saddle node bifurcation, which leads to an oscillatory behavior depending on the value of input $I_{a p p}$. Thus, the data-driven flow-map model must capture the oscillatory behavior for different values of $I_{a p p}$.

To train the PCK-based flow-map model, we generated one-step ahead samples of the states $V_{k}$ and $N_{k}$ by randomly drawing the initial states from $[-75,75] \times$ $[0,1]$. Here, we first examine the convergence error of the flow-map model to characterize how many samples of states would be necessary for data-driven reconstruction of the system dynamics. We quantify the convergence error in terms of the average MTE in Eq. (9) based on three validation trajectories generated for $I_{a p p}=$ $\{0,60,150\}$. Figure 3 shows the average MTE estimated over 1,000 time steps in relation to the number of training samples, where the vertical line around each 
error represents one standard deviation based on 5 repetitions of the analysis. It is evident that the error converges after about 160 samples, suggesting that a limited number of training samples is needed.

Figure 4 shows the reconstructed dynamics by the PCK-based flow-map model trained using 240 samples in comparison with the true dynamics. As can be seen, there is no visible discrepancy between the true timeevolution of the system and the reconstructed dynamics. The system exhibits a bifurcation behavior, as evident from the phase plots shown in Figure 4(c), (f), (i). Yet, the PCK-based flow-map model is able to capture this complex behavior and accurately predict the system dynamics over a long-time horizon. We note that a 500 -fold saving in the number of training samples is observed as compared to [23] in which a recurrent neural network representation was used for the flow-map model. This is while the PCK model also yields slightly more accurate predictions.

\subsection{Lorenz System}

We now consider a chaotic dynamical system based on the well-known Lorenz benchmark problem [59]. The Lorenz system has been widely used in the data-driven modeling literature (e.g., [60, 61]). The Lorenz system is described by the following set of nonlinear ordinary differential equations

$$
\begin{aligned}
& \frac{d a}{d t}=\sigma(b-a) \\
& \frac{d b}{d t}=a(\rho-c)-b \\
& \frac{d c}{d t}=a b-\beta c,
\end{aligned}
$$

where $s=[a, b, c]^{\top}$ are the system states and $\boldsymbol{x}=$ $[\sigma, \rho, \beta]^{\top}$ are the uncertain model parameters. Chaotic behaviors can be encountered in various chemical and biological systems, including in the growth of biological populations with non-overlapping generations [62] and the peroxidase-oxidase oscillator [63]. Here, we consider a constant time-lag $\delta=0.01$ that captures the intrinsic time-scale of the system [64].
The Lorenz system exhibits a chaotic behavior based on the initial conditions $\boldsymbol{s}_{0}$, while its long-term behavior is highly affected by the uncertain parameters $\boldsymbol{x}$. The nominal initial conditions and parameters of the system are, respectively, $s_{0}=[1.9427,-1.4045,0.9684]^{\top}$ and $\boldsymbol{x}_{0}=\left[\begin{array}{ll}10,28,8 / 3 \\ \top\end{array}\right]^{\top}$, for which the system oscillates around two attractors. Here, the training data consisted of 500 random samples of the state-space $s$ within the range $[-10,10] \times[-10,10] \times[-10,10]$ and the parameters $\boldsymbol{x}$ within the range $[8,12] \times[10,30] \times$ $[1,5.5]$. We used two validation trajectories to compare the true system dynamics with those reconstructed by the PCK-based flow-map model: one trajectory based on the nominal initial conditions and parameters and the other based on $\boldsymbol{x}=[10,15,8 / 3]^{\top}$ and $\boldsymbol{s}_{0}=$ $[1.6655,-0.1178,0.1748]^{\top}$.

Figure 5 shows phase plots of the reconstructed oscillatory dynamics of the Lorenz system, in comparison with the true system dynamics, over a simulation horizon of 5,000 time steps. We observe that the qualitative behavior of the Lorenz system is different when the parameter $\rho$ is varied, while the PCK-based flowmap model is able to reconstruct the dynamics in both cases. The MTE is 0.522 for the nominal validation trajectory and 0.0013 for the second validation trajectory. Although the error for the nominal validation trajectory seems relatively high, the main characteristics of the true dynamics are adequately captured, as evident from Figure 5(a)-(c). That is, the limit circles, the amplitude of oscillation and period are adequately captured. These predictions are consistent with those reported in [61]. However, we note that reconstruction of the Lorenz dynamics using neural networks typically requires on the order of a few thousands of training samples [20, 64], whereas the PCK model here was trained using 500 samples.

\section{3 | Transient Co-culture System}

In this case study, we demonstrate the ability of PCKbased flow-map models to learn the transient behavior of a co-culture system with variable inputs. In particular, we focus on the startup dynamics of a contin- 
(a)

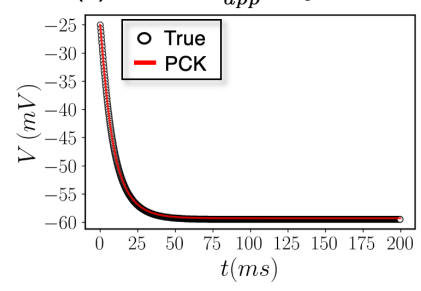

(d)

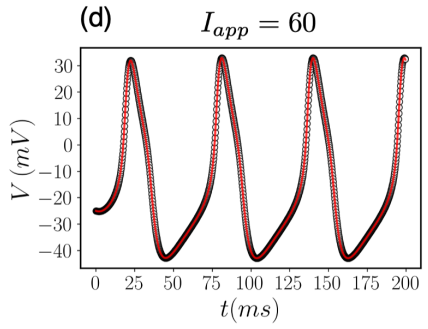

(g) $\quad I_{a p p}=150$

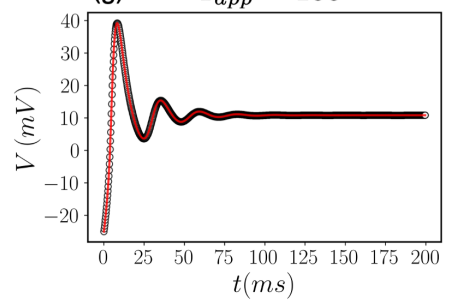

(b) $I_{a p p}=0$

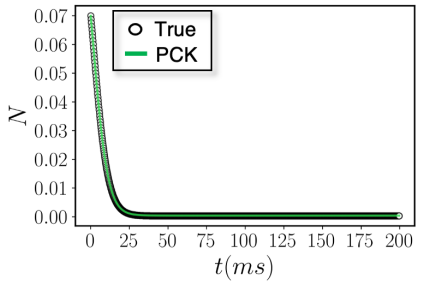

(e)

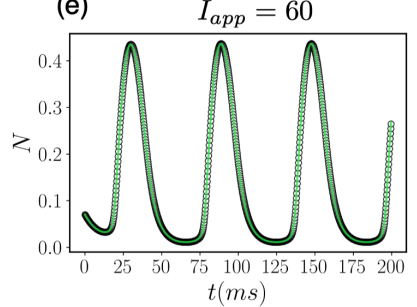

(h) $\quad I_{a p p}=150$

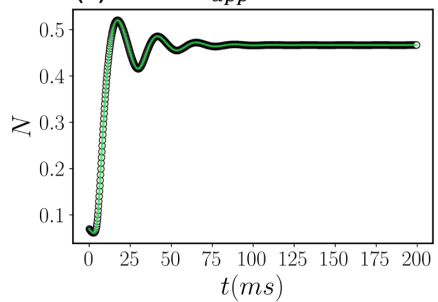

(c) $I_{a p p}=0$

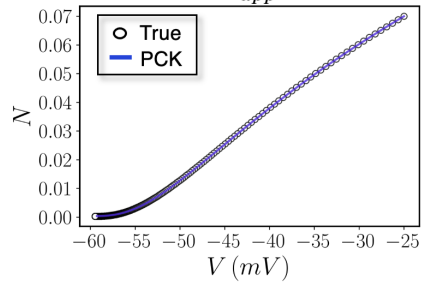

(f)

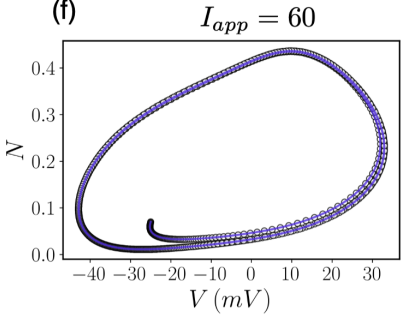

(i)

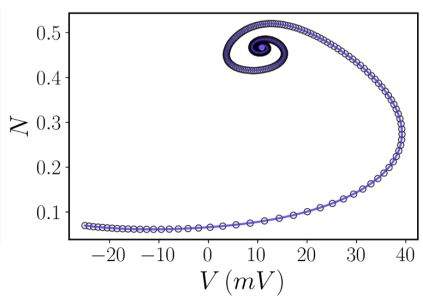

FIG URE 4 Reconstructed dynamics of the Morris-Lecar system by the PCK-based flow-map model in comparison with the true system dynamics for the input $I_{a p p}$ values $\{0,60,150\}$. The PCK-based flow-map model is trained using 240 samples. The left column shows the time-evolution of voltage difference, $V$; the middle column shows the time-evolution of the channel opening probability, $N$; and the right column shows the corresponding phase plots.

uous bioreactor driven by the competition of several auxotrophs [65]. To emulate data collection from a real system, we use a nonlinear dynamic model of the bioreactor [66] (given in the SI) to generate observations of the system states, which are then corrupted with independent and identically distributed state-dependent measurement noise $e_{i} \sim \mathcal{N}\left(0,2.5 \times 10^{-2} s_{k}^{i}\right)$, with $i$ being an index for the measured states and $k$ the time index. The five state variables $s_{k}$ of the bioreactor include: the population of the two species $N_{1}(\mathrm{Ce} / / \mathrm{s} / \mathrm{L})$ and $\mathrm{N}_{2}(\mathrm{Ce} / / \mathrm{s} / \mathrm{L})$, the auxotrophic nutrients concentrations $C_{1}(g / L)$ and $C_{2}(g / L)$, and the common shared carbon source concentration $C_{0}(g / L)$. The bioreactor has three process inputs $\boldsymbol{x}_{k}$ that can be varied in time. The process inputs are the dilution rate $D\left(\mathrm{hr}^{-1}\right)$ that varies within the range $[0.75,1.5]\left(\mathrm{hr}^{-1}\right)$, as well as the feed substrate concentration of auxotrophs $C_{1, i n}(\mathrm{~g} / \mathrm{l})$ and $C_{2, i n}$, both varying in the range $[1.5,2](\mathrm{g} / \mathrm{l})$. To generate data for training the PCK-based flow map models, short simulation "experiments" with a fixed length of $T=30$ steps with $\delta_{k} \in[0.15,0.25] \mathrm{hr}^{-1}$ were performed. At each time step $k$ during the multi-step experiments, inputs $\boldsymbol{x}_{k}$ were varied over the time interval $\delta_{k}$ and noisy observations of the states were collected.

For the validation plots of Figure 6, we begin by some random initial condition at $k=0$, by applying an input $x_{0}$ over some interval $\delta_{0}$. The model predicts the mean of the states at $k=1$, as well as their variance. The integration proceeds by taking a next step based on the mean value of the states at $k=1$, predicting 
(a)

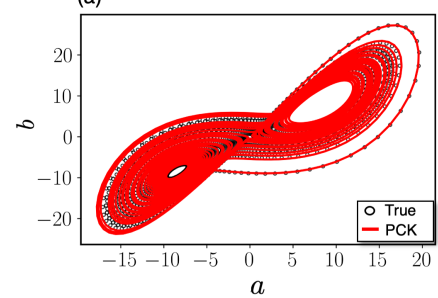

(d)

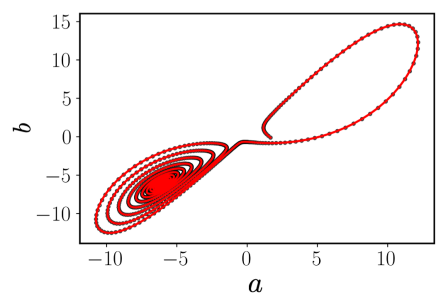

(b)

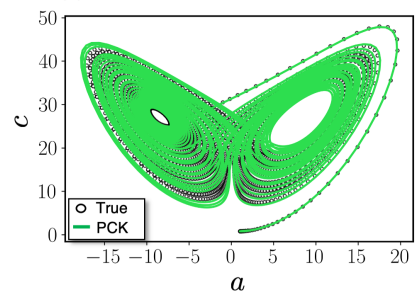

(e)

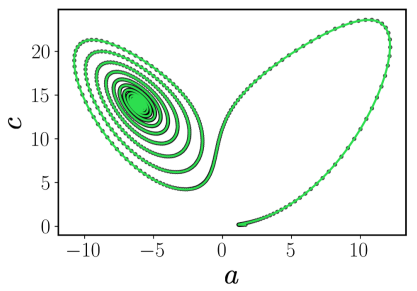

(c)

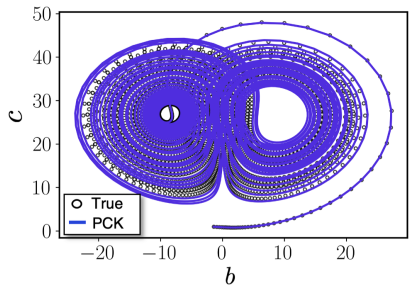

(f)

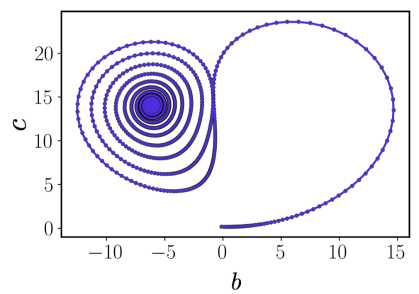

FIG URE 5 Phase plots of the reconstructed dynamics of the Lorenz system by the PCK-based flow-map model in comparison with the true system dynamics for different values of model parameters. Subplots (a)-(c) correspond to the model parameters $\sigma=10, \beta=8 / 3$, and $\rho=28$. Subplots (d)-(f) correspond to the model parameters $\sigma=10, \beta=8 / 3$, and $\rho=15$.

the states at $k=2$. Using only the mean value to compute trajectories is probably the simplest way when Gaussian Process state space models are utilized, however, there are more sophisticated ways for the trajectory generation [67], which are beyond the scope of the paper. Note that properly incorporating the uncertainty in multi-step ahead predictions is a complicated issue addressed in the literature $[68,69]$. Here, it suffices to use a deterministic function, e.g., the mean value of the data-driven flow-map model, to integrate in time since this way we avoid the major issue of using noisy inputs into our PCK model. The validation trajectories have a length of $N_{k}=40$ steps ahead, extending slightly beyond the training range. Moreover, thanks to the nature of the PCK model, we can also simply characterize the confidence of the model to the prediction of the dynamics. To get some uncertainty estimates on the predicted trajectories, at each step $k$, we plot the $3 \sigma\left(w_{k}\right)$ error bars around the mean. Overall, we observe that the true, noiseless trajectories are embedded within the confidence intervals of the PCK predictions.

\section{4 | UNCERTAINTY QUANTIFICA- TION OF EXPENSIVE COMPUTA- TIONAL MODELS}

In this section, we demonstrate the utility of data-driven flow-maps for the UQ of a Microbial Electrosynthesis (MES) bioreactor using a high-fidelity computational model that is subject to uncertainty in model parameters and initial conditions. In particular, we show how flow-maps can be used as surrogate models for efficient sample-based approximation of distribution of Qols, global sensitivity analysis, and Bayesian parameter inference, when the original model is prohibitively expensive for a sample-based analysis.

We consider the batch MES bioreactor shown in Figure 7 for $\mathrm{CO}_{2}$ fixation [70], with potential applications in space biomanufacturing [71]. The bioreactor consists of a well-mixed liquid bulk phase that contains dissolved $\mathrm{CO}_{2}$, i.e., substrate. A microbial community forming a biofilm grows on the cathode of the bioreactor. The dissolved substrate diffuses into the biofilm through a linear boundary layer and is then consumed by bacteria towards the growth of the biofilm. This leads to spa- 
tial distribution of the substrate concentration within the biofilm. Voltage is applied to the cathode while the biofilm acts as a conductive matrix through which electron transport takes place. Both the substrate $\mathrm{CO}_{2}$ in the biofilm and the local overpotential due to the current flux contribute to the biofilm growth kinetics described by the dual Monod-Nerst model [72].

A computational model of the dynamics of the MES bioreactor is adopted from [73, 74], with some modifications. Within the biofilm, the cell growth leads to the production of acetate as a metabolic product. A primary modeling approach in the aforementioned papers assumes the total biomass has a constant concentration and exists in two forms, active and inactive, each of which occupies some volume fraction. We assume that biomass exists only in active form, thus the equations describing the volume-fraction change within the film effectively become a single equation for the rate of change of film thickness, $L_{f}$, which is a differential state in our system. Moreover, the film growth is affected by a constant detachment rate. It is also assumed that the reaction occurs only within the biofilm, so the only source of acetate in the bulk phase comes from exchange with the biofilm through the boundary layer. We further assume the transport-reaction phenomena in the biofilm are much faster than the transport that occurs across the boundary layer and in the bulk phase; accordingly, the conservation laws inside the biofilm are considered to be in pseudo steady-state [73]. Hence, the computational model consists of a set of nonlinear secondorder ordinary differential equations that describe the spatial distribution of substrate, acetate and overpotential within the biofilm, coupled with a set of first-order ordinary differential equations that describe the concentration of $\mathrm{CO}_{2}$ in the bulk phase $S_{b}$, the acetate concentration in the bulk phase $P_{b}$, and the biofilm thickness (a)

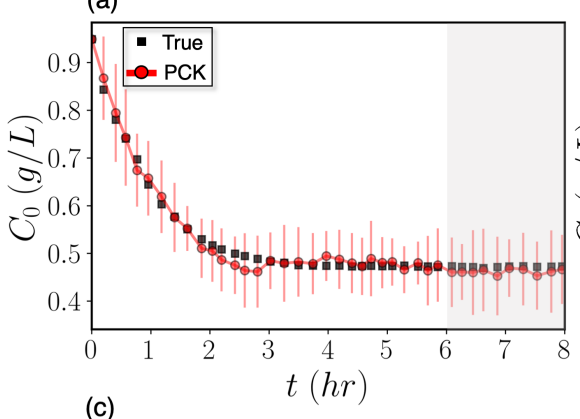

(c)

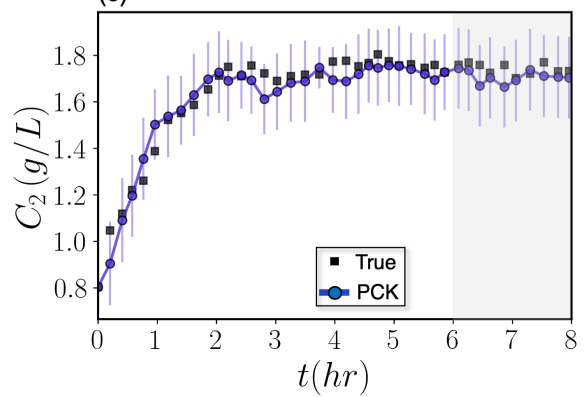

(b)
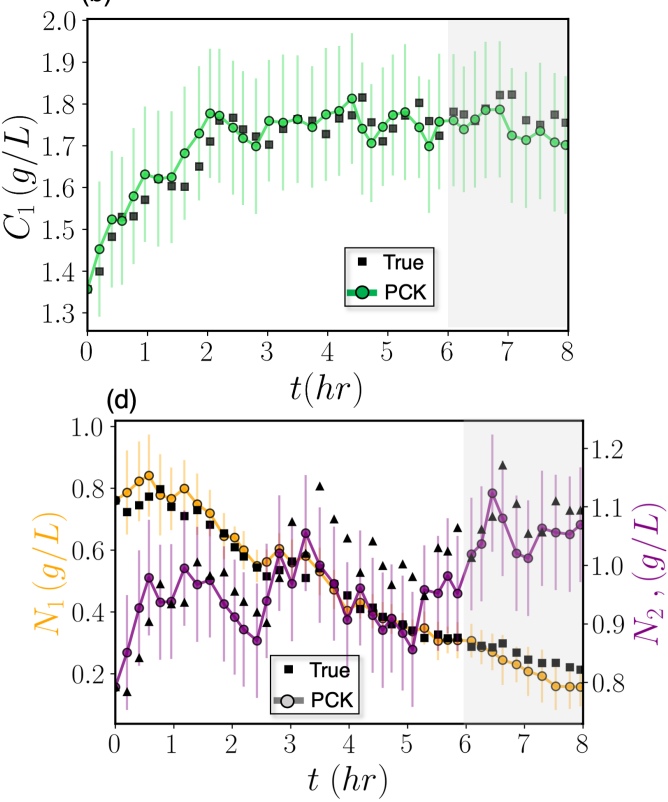

FIG URE 6 Predictions of the state variables of the transient co-culture system via the PCK-based flow-map models in comparison with the observed state trajectories. The colored lines/points correspond to the predicted trajectories by the mean of the PCK models, starting from some initial states at $t=0 \mathrm{hr}$. Black symbols represent the observed trajectories at specific snapshots during a validation run. Vertical error bars represent the uncertainty in the predictions of the PCK models, estimated as plus/minus two standard deviations from the mean value. The shaded areas correspond to a time interval that was not accounted for when training the PCK models. 
$L_{f}$. As such, the three state variables of the system are described by

$$
\begin{aligned}
\frac{d L_{f}}{d t} & =\left(Y \hat{q}-r_{d}\right) L_{f} \\
\frac{d S_{b}}{d t} & =\frac{A_{f}}{V_{r}} j S \\
\frac{d P_{b}}{d t} & =\frac{A_{f}}{V_{r}} j P,
\end{aligned}
$$

where $Y\left(\frac{m g X}{m m o / S}\right)$ is the biomass yield coefficient, $\hat{q}\left(\frac{\mathrm{mmol} S}{m g X \text { days }}\right)$ represents an average substrate consumption specific rate within the biofilm, $r_{d}\left(\frac{1}{\text { days }}\right)$ is a detachment rate, $A_{f}\left(\mathrm{~cm}^{2}\right)$ is the cross-sectional area of the biofilm, and $V_{r}\left(\mathrm{~cm}^{3}\right)$ is the bioreactor volume. The mass balances for the substrate and product are a function of the flux of each species across the linear boundary layer described by

$$
j_{m}=\frac{D_{b}}{L_{b}}\left(m_{f}\left(z=L_{f}\right)-m_{b}\right), \quad m=S, P
$$

where $m$ denotes the species (i.e., substrate and product), $D_{b}\left(\frac{\mathrm{cm}^{2}}{\text { days }}\right)$ is the diffusivity coefficient in the boundary layer and $L_{b}(\mathrm{~cm})$ is the thickness of the boundary layer. The subscript $f$ denotes the species concentra-

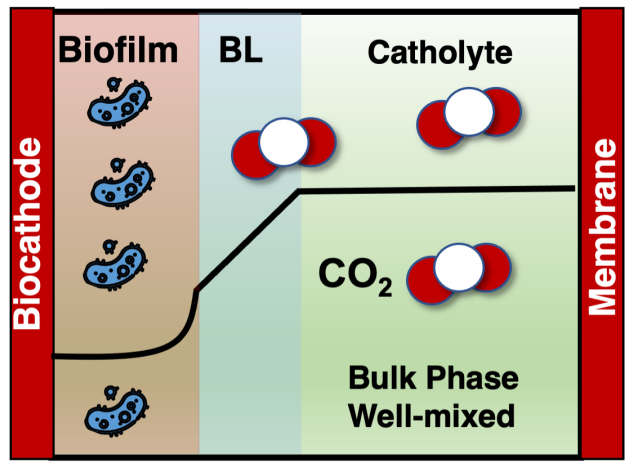

FIGURE 7 Schematic of the microbial electrosynthesis bioreactor. The bioreactor consists of 3 regions: the bulk phase, the biofilm, and a boundary layer (BL) in between. The black line represents a typical concentration profile of some species as predicted by the computational model used in this work. The concentration is assumed to be constant in the bulk phase, changing linearly across the boundary layer, and exhibiting a more complicated shape in the biofilm. tion in the film at position $z=L_{f}$. The equations that $\quad 591$ describe the diffusion phenomena within the film are given in the SI. In order to determine the concentrations at $L_{f}$, a boundary value problem (diffusion within the film) must be solved at each time step, as the concentrations in the biofilm are a function of the bulk concentrations. The computational model is fairly expensive for UQ analyses that rely on Monte Carlo sampling; each model run takes on average 4.5 minutes. The model is subject to time-invariant uncertainty in its parameters and initial conditions. Specifically, the model uncertainty comprises of the conductivity of the biofilm $k_{\text {bio }}$, the maximum growth rate $\mu_{\max }$ of the Nerst-Monod model, the yield $Y$, the Monod affinity constant $K_{s}$, as well as the acetate production-related parameters $\alpha$ and $\beta$. These six uncertain parameters are assumed to follow a uniform probability distribution. Their nominal values are $\left[k_{\text {bio }}, \mu_{\max }, Y, K_{s}, \alpha, \beta\right]^{\top}=[1 \times$ $\left.10^{-3}, 4.5,0.25,3.0,0.1,2 \times 10^{-5}\right]^{\top}$, while they vary uniformly $\pm 10 \%$ from the nominal values.

In this case study, we construct data-driven flowmap models of the PCK form in Eq. (5) for the Qols $\boldsymbol{y}=$ $\left[\begin{array}{lll}L_{f} & S_{b} & P_{b}\end{array}\right]^{\top}$, such that the six sources of uncertainty constitute the vector of input variables $\boldsymbol{x}$ in Eq. (5). The three flow-map models, one for each Qol, were trained using simulation data generated via the computational model for lag times in the range of $\delta=[0.05,0.1]$ days, which allow us to adequately capture the bioreactor dynamics. Notice that clearly the lag time $\delta$ must always be larger than the integration time step of the computational model.

The training dataset consists of full state trajectories, as well as one-step ahead samples of the states. We initially generate $N_{T}=30$ trajectories, with fixed uncertain parameters in time, over a process time span from 0 to 3.5 days, which corresponds to approximately $T=50$ samples per trajectory. Then, using the states $s_{k}$ corresponding to each sample $\boldsymbol{w}_{k}$, we randomize the uncertain parameters and perform one-step ahead simulations. In this way, approximately 1,400 training samples were generated, while 800 samples are used for training the PCK models. The rationale behind not randomizing the states is that the validation trajectories (step 
0 of Figure 2) indicate that there is a high correlation among state values. For instance, as $L_{f}$ grows in time (under insignificant detachment), $s_{b}$ decreases due to consumption. Thus, for a given set of uncertain parameters and initial states, a few full state trajectories will help generate more informative training samples. Figure 8 shows the predicted trajectories using the datadriven flow-map PCK model for a given realization of uncertainty and initial conditions, while the true trajectory is juxtaposed. The trajectories correspond to a timemarch of 50 steps ahead. We observe a perfect agreement between the predicted and validation trajectories, with the average MTE for the three states being approximately $\hat{\epsilon}=2.5 \times 10^{-4}$.

An important remark should be made here regarding the benefits of the presented flow-map approach to surrogate modeling of dynamical systems in comparison with the so-called time-frozen approaches discussed in Section 1. First, the flow-map models provide the flexibility to approximate the distribution of states at any time instant of interest without the need for constructing a separate surrogate model for each time instant, as in time-frozen surrogate modeling. For example, if we were to use a time-frozen approach, 50 separate PCK models would need to be constructed for each Qol to predict the time-evolution of the Qol distribution over the 50 time instants considered here. Thus, not only a flow-map modeling approach significantly reduces the number of surrogate models that must be constructed to only one model for each Qol, it also provides flexibil- ity via alleviating the need to build the models at prespecified time points. Furthermore, the flow-map modeling approach enables more efficient data generation. To clarify this point, let us assume that $N_{p}$ realizations of uncertainty are sufficient for generating a rich training dataset that yields surrogate models with low approximation error. In the case of the time-frozen approach, we would require to generate $N_{p}$ full state trajectories since the states must be observed at all time instants for all uncertainty realizations. This approach to data generation can become prohibitively expensive, in particular when data generation relies on expensive simulations. However, training the flow-map models, in principle, requires simulation of a limited number of full state trajectories (in this study, 25 trajectories), whereas $N_{p}$ training samples can be straightforward generated via one-step ahead integration of the computational model. In the following, the use of PCK-based flow-map models is demonstrated for expensive UQ analyzes.

\subsection{Forward Uncertainty Propagation and Global Sensitivity Analysis}

Here, we use the data-driven flow-map models for efficient uncertainty propagation via sample-based approximation of the distribution of the three Qols. Figure 9(a)(c) shows the distribution of the Qols at $t=3.5$ days. To approximate the distribution of Qols, the flow-map models were evaluated using 20,000 realizations of the model uncertainty $\boldsymbol{x}$. Each run of the data-driven flow-
664

665

666

667

668

669

670

671

672

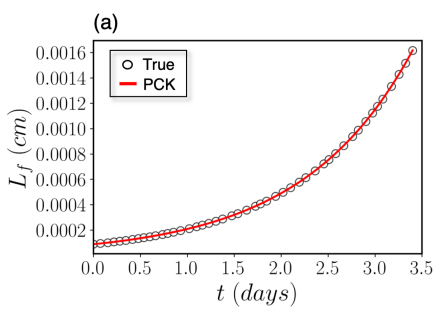

(b)

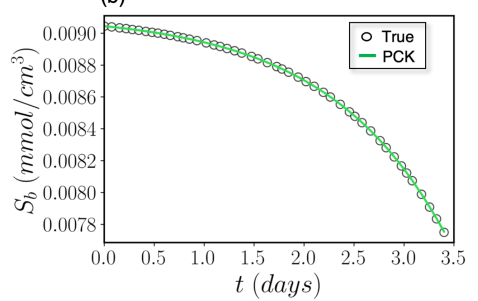

(c)

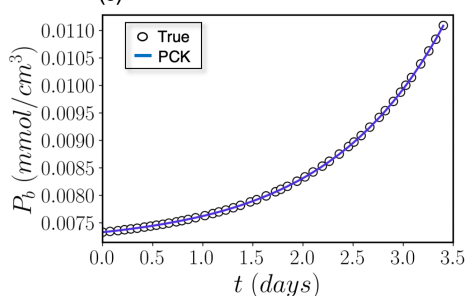

FIGURE 8 Predicted state trajectories of the the microbial electrosynthesis bioreactor: (a) biofilm thickness, $L_{f}$, (b) $\mathrm{CO}_{2}$ concentration in the bulk phase, $S_{b}$, and (c) acetate concentration in the bulk phase, $P_{b}$. Hollow points represent the validation trajectories, while the solid lines represent the trajectories predicted by the PCK-based flow-map models. 
(a)

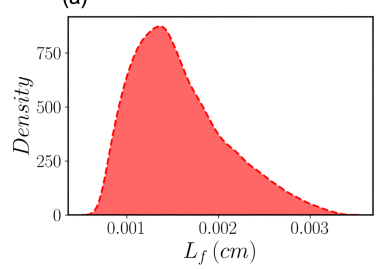

(d)

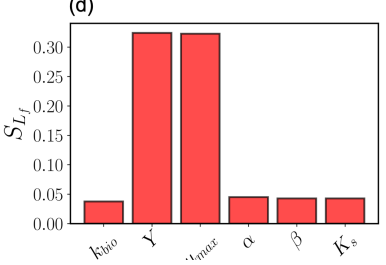

(b)

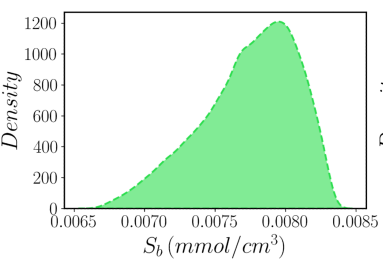

(e)

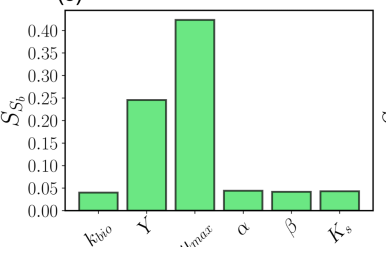

(c)

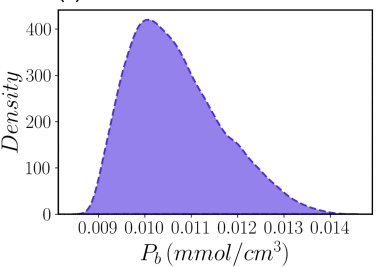

(f)

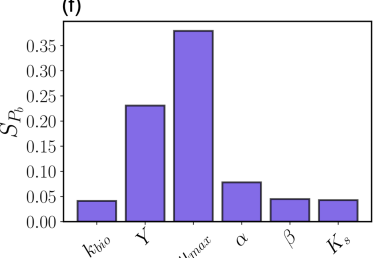

FIG URE 9 Fast uncertainty propagation and global sensitivity analysis of the the microbial electrosynthesis bioreactor using data-driven flow-map models of quantities of interest. Subplots (a)-(c) show the kernel density estimates of the distribution of the biofilm thickness $\left(L_{f}\right)$, concentration of $\mathrm{CO}_{2}$ in the bulk phase $\left(S_{b}\right)$, and acetate concentration in the bulk phase $\left(P_{b}\right)$ predicted by the PCK models at time $t=3.5$ days. The distributions of $L_{f}, S_{b}$ and $P_{b}$ are approximated via Monte Carlo sampling using 20,000 realizations of uncertain model parameters, where a 100-fold computational speedup in sample-based approximation of the distributions is attained. Subplots (d)-(f) show the Borgonovo indices, denoted by $\mathcal{S}$, that quantify the global sensitivity of $L_{f}, S_{b}$ and $P_{b}$ at $t=3.5$ days with respect to the six uncertain model parameters. The Borgonovo indices are approximated based on 20,000 uncertainty realizations.

\footnotetext{
${ }^{1}$ Notice that the evaluation time of a PCK model depends on a multitude of factors, such as the degree of the polynomial basis functions, kernel type, and, mainly, amount of data used to train the model. Additionally, a kernel-based model such as PCK is more expensive to evaluate than a polynomial chaos expansion.
}

gonovo indices [75], denoted by $\mathcal{S}$, which are based on the full distribution of Qols, as opposed to their statistical moments. The results of global sensitivity analysis of Qols at $t=3.5$ days are shown in Figure 9(d)-(f), where each bar corresponds to a different uncertain model parameter. The Borgonovo indices are approximated using the same 20,000 samples used in forward UQ. We observe that the probabilistic uncertainty of yield $Y$ and maximum growth rate $\mu_{\max }$ have the most dominant effects on the variability of the three Qols, while the product concentration $P_{b}$ is also significantly affected by the uncertainty in the parameter $\alpha$, which is the metabolismrelated productivity constant.

\section{2 | Bayesian Inference of Unknown Model Parameters}

We now use the data-driven flow-map models to solve a Bayesian inference problem in order to infer the uncertain model parameters $\boldsymbol{x}$. Bayesian inference relies on Bayes theorem to estimate the posterior probability distribution of the unknown model parameters from 
(a)

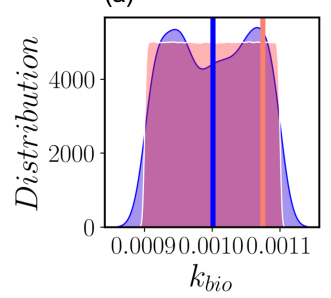

(d)

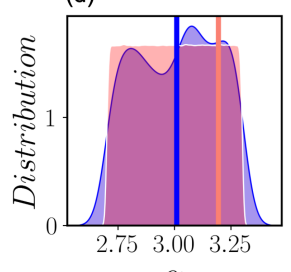

(b)

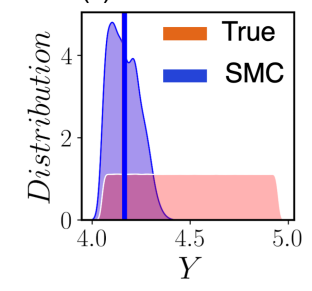

(e)

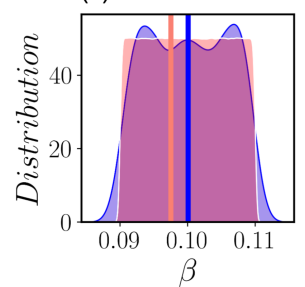

(c)

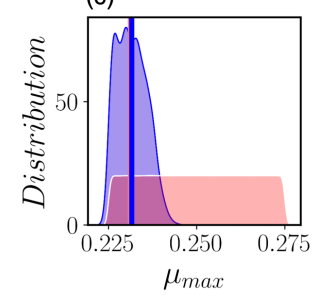

(f)

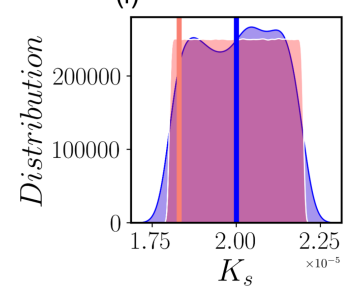

FIGURE 10 Bayesian inference of unknown parameters of the computational model of the microbial electrosynthesis bioreactor. The parameters are estimated via sequential Monte Carlo using 20,000 particles. Red and blue distributions represent the prior and posterior distributions of the unknown model parameters at time 3.5 days, respectively. The red vertical lines correspond to the true parameters, while the blue vertical lines are the estimated posterior mean value of parameters.

available data. Here, noisy observations of $L_{f}, S_{b}$ and $P_{b}$ at time instants $\{0.5,1.0,1.5,2.0,2.5,3.0,3.5\}$ days constitute the dataset $\mathcal{D}$ used for parameter inference; measurement noise is modeled as a Gaussian distribution with zero mean and state-dependent variance. Once a vector of system measurements $\boldsymbol{d}$ at a time instant is observed, the change in our knowledge about the unknown parameters is described by Bayes' rule [76]

$$
f_{\boldsymbol{x} \mid \mathcal{D}}(\boldsymbol{x} \mid \boldsymbol{d})=\frac{f_{\mathcal{D} \mid \boldsymbol{x}}(\boldsymbol{d} \mid \boldsymbol{x}) f_{\boldsymbol{x}}(\boldsymbol{x})}{f_{\mathcal{D}}(\boldsymbol{d})},
$$

where $f_{x \mid \mathcal{D}}$ denotes the posterior distribution of the uncertain parameters after observing the data; $f_{\mathcal{D} \mid x}$ is the likelihood function that describes the probability of observing data given the parameter estimates; $f_{\boldsymbol{x}}$ is the prior distribution of parameters; and $f_{\mathcal{D}}$ is the so-called evidence or marginal likelihood that ensures the posterior distribution integrates to 1.

As Eq. (14) implies, Bayesian inference provides an explicit representation of the uncertainty in the parameter estimates via characterizing the full posterior dis- tribution of unknown parameters $x$. The prior distribution of parameters and the likelihood function must be specified to solve Eq. (14). Here, we used the same uniform distributions as those used to construct the PCK surrogate models to represent the prior distributions, although these can be different. The likelihood function is specified by the observation noise model, which is assumed to be zero-mean Gaussian with state-dependent variance in this work. We use a particle filtering method, namely sequential Monte Carlo (SMC) [77], to approximately solve the Bayesian inference problem by iteratively updating the posterior $f_{\boldsymbol{x} \mid \mathcal{D}}$ at every time instant that system observations become available; see [43] for further details. Notice that parameter estimation via Bayesian inference methods such as SMC relies on accurate construction of the probability distributions in Eq. (14). As described in Section 4.1, the data-driven flow-map models enable efficient sample-based approximation of the distributions using a very large number of samples, which otherwise could be impractical using an expensive computational model.

Figure 10 shows the posterior distribution of the pa- 
rameters $\boldsymbol{x}$ at $t=3.5$ days estimated via SMC using the dataset $\mathcal{D}$, as specified above. The posterior distributions are approximated using 20,000 particles. Note that the posterior distribution ranges seem to be larger than the prior in some cases, which is an artifact of the kernel density estimation (i.e., the selection of the bandwidth parameter) [78]. Figure 10 suggests that only the posterior distributions of parameters $Y$ and $\mu_{\max }$ have changed significantly with respect to their priors. It is also evident that the mean of the posterior distributions (blue vertical lines) for parameters $Y$ and $\mu_{\max }$ provides a fairly accurate estimate for the true, but unknown, parameter values (brown vertical lines). In particular, the true value and the posterior mean are indistinguishable, while the posteriors are much more narrow compared to priors as stated before. Nonetheless, the posterior distributions for the other parameters remain similar to their priors with little to no change, suggesting these parameters cannot be estimated using the available dataset $\mathcal{D}$. This can be attributed to the lack of information content of system observations $\mathcal{D}$ for inferring the unknown parameters; a deficiency that can be addressed via optimal experiment design [79, 80]. We again note the flexibility of the flow-map models that would allow us to seamlessly add new observation points, should that become necessary for better parameter inference, without the need to construct new surrogate models for the Qols observed at new time points.

\section{5 | CONCLUSIONS}

This paper presented a flow-map modeling approach based on polynomial chaos Kriging for the discovery of system dynamics from data. Data-driven flow-map models directly approximate the integration operator of differential equations that describe the state transitions of a dynamical system as a function of system state and input variables. We illustrated the usefulness of the proposed approach for learning mathematical descriptions of nonlinear dynamical systems and deriving dynamic surrogate models for fast uncertainty quantification applications. Our analyzes reveal that polynomial chaos Kriging-based flow-maps offer significant bene- $\quad \mathbf{8 1 0}$ fits in terms of data efficiency, as well as computational efficiency of data generation, for the discovery of nonlinear system dynamics and surrogate modeling.

\section{Data Availability}

814

All software required for reproducing the case studies presented is available through the CUBES github organization at https://github.com/cubes-space/ DataDriven-FlowMaps and any additional data is available upon request.

\section{Authorship Contributions}

GM and $A M$ conceived the concept of this contribution. GM performed the analysis with help from AJB and $F S$ and oversight from AM, APA, and DSC. GM, AM, and AJB wrote the manuscript. All authors edited the manuscript.

\section{Competing Interests}

The authors declare that they have no conflicts of interest.

\section{Acknowledgements}

This material is based upon work supported by NASA under grant or cooperative agreement award number NNX17AJ31G.

\section{references}

[1] Julio R Banga, Eva Balsa-Canto, Carmen G Moles, and Antonio A Alonso. Dynamic optimization of bioprocesses: Efficient and robust numerical strategies. Journal of Biotechnology, 117(4):407-419, 2005.

[2] Gaia Franceschini and Sandro Macchietto. Modelbased design of experiments for parameter precision: State of the art. Chemical Engineering Science, 63(19): 4846-4872, 2008.

[3] Andrew Golightly and Darren J Wilkinson. Bayesian parameter inference for stochastic biochemical net-

1

15

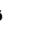


work models using particle markov chain monte carlo. Interface focus, 1(6):807-820, 2011.

[4] Bertrand looss and Paul Lemaître. A review on global sensitivity analysis methods. In Uncertainty management in simulation-optimization of complex systems, pages 101-122. Springer, 2015.

[5] Michael D Schmidt, Ravishankar R Vallabhajosyula, Jerry W Jenkins, Jonathan E Hood, Abhishek S Soni, John P Wikswo, and Hod Lipson. Automated refinement and inference of analytical models for metabolic networks. Physical biology, 8(5):055011, 2011.

[6] Bryan C Daniels and Ilya Nemenman. Efficient inference of parsimonious phenomenological models of cellular dynamics using s-systems and alternating regression. PloS one, 10(3):e0119821, 2015.

[7] S Feyo De Azevedo, B Dahm, and FR Oliveira. Hybrid modelling of biochemical processes: A comparison with the conventional approach. Computers \& Chemical Engineering, 21:S751-S756, 1997.

[8] Moritz Von Stosch, Rui Oliveira, Joana Peres, and Sebastião Feyo de Azevedo. Hybrid semi-parametric modeling in process systems engineering: Past, present and future. Computers \& Chemical Engineering, 60:86-101, 2014.

[9] Jörg Schubert, Rimvydas Simutis, Michael Dors, Ivo Havlik, and Andreas Lübbert. Bioprocess optimization and control: Application of hybrid modelling. Journal of biotechnology, 35(1):51-68, 1994.

[10] Dongda Zhang, Ehecatl Antonio Del Rio-Chanona, Panagiotis Petsagkourakis, and Jonathan Wagner. Hybrid physics-based and data-driven modeling for bioprocess online simulation and optimization. Biotechnology and bioengineering, 116(11):2919-2930, 2019.

[11] Josh Bongard and Hod Lipson. Automated reverse engineering of nonlinear dynamical systems. Proceedings of the National Academy of Sciences, 104(24):99439948, 2007.

[12] Kathleen Champion, Bethany Lusch, J Nathan Kutz, and Steven L Brunton. Data-driven discovery of coordinates and governing equations. Proceedings of the National Academy of Sciences, 116(45):22445-22451, 2019.

[13] Steven L Brunton, Joshua L Proctor, and J Nathan Kutz. Discovering governing equations from data by sparse identification of nonlinear dynamical systems. Proceedings of the national academy of sciences, 113(15): 3932-3937, 2016.
[14] Markus Heinonen, Cagatay Yildiz, Henrik Mannerström, Jukka Intosalmi, and Harri Lähdesmäki. Learning unknown ode models with gaussian processes. In International Conference on Machine Learning, pages 1959-1968. PMLR, 2018.

[15] J Nathan Kutz, Steven L Brunton, Bingni W Brunton, and Joshua L Proctor. Dynamic mode decomposition: data-driven modeling of complex systems. SIAM, 2016.

[16] Peter J Schmid. Dynamic mode decomposition of numerical and experimental data. Journal of fluid mechanics, 656:5-28, 2010.

[17] Matthew O Williams, loannis G Kevrekidis, and Clarence W Rowley. A data-driven approximation of the koopman operator: Extending dynamic mode decomposition. Journal of Nonlinear Science, 25(6):13071346, 2015.

[18] Johan Schoukens and Lennart Ljung. Nonlinear system identification: A user-oriented road map. IEEE Control Systems Magazine, 39(6):28-99, 2019.

[19] Maziar Raissi, Paris Perdikaris, and George E Karniadakis. Physics-informed neural networks: A deep learning framework for solving forward and inverse problems involving nonlinear partial differential equations. Journal of Computational Physics, 378:686-707, 2019.

[20] Samuel H Rudy, J Nathan Kutz, and Steven L Brunton. Deep learning of dynamics and signal-noise decomposition with time-stepping constraints. Journal of Computational Physics, 396:483-506, 2019.

[21] Tong Qin, Kailiang Wu, and Dongbin Xiu. Data driven governing equations approximation using deep neural networks. Journal of Computational Physics, 395:620635, 2019.

[22] Tong Qin, Zhen Chen, John Jakeman, and Dongbin Xiu. Deep learning of parameterized equations with applications to uncertainty quantification, 2020.

[23] Wei-Hung Su, Ching-Shan Chou, and Dongbin Xiu. Deep Learning of Biological Models from Data: Applications to ODE Models. Bulletin of Mathematical Biology, 83(3):1-19, 2021.

[24] Stefan Streif, Kwang Ki K Kim, Philipp Rumschinski, Masako Kishida, Dongying Erin Shen, Rolf Findeisen, and Richard D Braatz. Robustness analysis, prediction, and estimation for uncertain biochemical networks: An overview. J. Process Control, 42:14-34, 2016. ISSN 09591524. doi: 10.1016/j.jprocont.2016.03.004. 
[25] Habib N Najm. Uncertainty Quantification and Polynomial Chaos Techniques in Computational Fluid Dynamics. Annual Review of Fluid Mechanics, 41(1):35-52, 2009. doi: 10.1146/annurev.fluid.010908.165248.

[26] Ralph C Smith. Uncertainty quantification: theory, implementation, and applications, volume 12. Siam, 2013.

[27] Michał Komorowski, Bärbel Finkenstädt, Claire V Harper, and David A Rand. Bayesian inference of biochemical kinetic parameters using the linear noise approximation. BMC bioinformatics, 10(1):1-10, 2009.

[28] Philipp Rumschinski, Steffen Borchers, Sandro Bosio, Robert Weismantel, and Rolf Findeisen. Set-base dynamical parameter estimation and model invalidation for biochemical reaction networks. BMC systems biology, 4(1):1-14, 2010.

[29] J Vanlier, C A Tiemann, P A J Hilbers, and N A W Van Riel. Parameter uncertainty in biochemical models described by ordinary differential equations. Mathematical biosciences, 246(2):305-314, 2013.

[30] Ali Mesbah and Stefan Streif. A probabilistic approach to robust optimal experiment design with chance constraints. IFAC-PapersOnLine, 48(8):100-105, 2015.

[31] Joel A Paulson, Marc Martin-Casas, and Ali Mesbah. Fast uncertainty quantification for dynamic flux balance analysis using non-smooth polynomial chaos expansions. PLoS computational biology, 15(8): e1007308, 2019.

[32] Russel E Caflisch. Monte Carlo and Quasi-Monte Carlo methods. Acta numerica, 7:1-49, 1998.

[33] B Sudret, S Marelli, and J Wiart. Surrogate models for uncertainty quantification: An overview. In 201711 th European Conference on Antennas and Propagation (EUCAP), pages 793-797, 3 2017. doi: 10.23919/EuCAP. 2017.7928679.

[34] Dongbin Xiu and George Em Karniadakis. Modeling uncertainty in flow simulations via generalized polynomial chaos. Journal of Computational Physics, 187 (1):137-167, 5 2003. ISSN 00219991. doi: 10.1016/ S0021-9991(03)00092-5.

[35] Géraud Blatman and Bruno Sudret. Adaptive sparse polynomial chaos expansion based on least angle regression. Journal of computational Physics, 230(6): 2345-2367, 2011.

[36] Noel Cressie. The origins of kriging. Mathematical geology, 22(3):239-252, 1990.
[37] Rohit K Tripathy and Ilias Bilionis. Deep UQ: Learning deep neural network surrogate models for high dimensional uncertainty quantification. Journal of Computational Physics, 375:565-588, 2018. ISSN 00219991. doi: https://doi.org/10.1016/j.jcp.2018.08. 036. URL http://www.sciencedirect.com/science/ article/pii/S0021999118305655.

[38] Stefan Streif, Felix Petzke, Ali Mesbah, Rolf Findeisen, and Richard D Braatz. Optimal experimental design for probabilistic model discrimination using polynomial chaos. IFAC Proceedings Volumes, 47(3):41034109, 2014.

[39] Ehecatl Antonio del Rio-Chanona, Jonathan L Wagner, Haider Ali, Fabio Fiorelli, Dongda Zhang, and Klaus Hellgardt. Deep learning-based surrogate modeling and optimization for microalgal biofuel production and photobioreactor design. AIChE Journal, 65(3):915923, 2019.

[40] Claudia Schillings, Mikael Sunnåker, Jörg Stelling, and 1000 Christoph Schwab. Efficient characterization of para- 1001 metric uncertainty of complex (bio) chemical net- 1002 works. PLoS Computational Biology, 11(8):e1004457, 1003 2015.

[41] Fábio Henrique Pereira, Pedro HT Schimit, and Fran- 1005 cisco Elânio Bezerra. A deep learning based surro- 1006 gate model for the parameter identification problem 1007 in probabilistic cellular automaton epidemic models. 1008 Computer Methods and Programs in Biomedicine, 205: 1009 106078, 2021.

[42] CL Pettit and PS Beran. Spectral and multiresolu- 1011 tion wiener expansions of oscillatory stochastic pro- 1012 cesses. Journal of Sound and Vibration, 294(4-5):752- 1013 779, 2006.

[43] Georgios Makrygiorgos, Giovanni Maria Maggioni, 1015 and Ali Mesbah. Surrogate modeling for fast uncer- 1016 tainty quantification: Application to $2 \mathrm{~d}$ population bal- 1017 ance models. Computers \& Chemical Engineering, 138: 1018 106814, 2020.

[44] R Schöbi and Bruno Sudret. PC-Kriging: a new meta- 1020 modelling method combining polynomial chaos expan- 1021 sions and kriging. Proc. 2nd Int. Symp. Uncertain. Quan- 1022 tif. Stoch. Model., 2014.

[45] Georgios Makrygiorgos, Soumyajit Sen Gupta, Amor A 1024 Menezes, and Ali Mesbah. Fast probabilistic uncer- 1025 tainty quantification and sensitivity analysis of a mars 1026 life support system model. IFAC-PapersOnLine, 53(2): 1027 7268-7273, 2020.

\section{3}

\section{4}

95

\section{2}

86

\section{7}

89

991 996
997

998 
[46] G Deman, K Konakli, B Sudret, J Kerrou, P Perrochet, and $\mathrm{H}$ Benabderrahmane. Using sparse polynomial chaos expansions for the global sensitivity analysis of groundwater lifetime expectancy in a multi-layered hydrogeological model. Reliability Engineering and System Safety, 147:156-169, 2016. ISSN 09518320.

[47] Joel A Paulson, Marc Martin-Casas, and Ali Mesbah. Optimal Bayesian experiment design for nonlinear dynamic systems with chance constraints. Journal of Process Control, 77:155-171, 2019. ISSN 0959-1524. doi: https://doi.org/10.1016/j.jprocont.2019.01.010.

[48] S Oladyshkin and W Nowak. Data-driven uncertainty quantification using the arbitrary polynomial chaos expansion. Reliab. Eng. Syst. Saf., 106:179-190, 2012. ISSN 09518320. doi: 10.1016/j.ress.2012.05.002.

[49] K I Williams and C Rasmussen. Gaussian processes for machine learning. MIT Press, 2006. ISBN 026218253X. doi: 10.1142/S0129065704001899.

[50] Dongbin Xiu and George Em Karniadakis. The Wiener-Askey Polynomial Chaos for Stochastic Differential Equations. SIAM J. Sci. Comput., 24(2):619644, 1 2002. ISSN 1064-8275. doi: 10.1137/ S1064827501387826.

[51] Author R H Cameron and W T Martin. Annals of Mathematics The Orthogonal Development of Non-Linear Functionals in Series of Fourier-Hermite Functionals. Ann. Math., 48(2):385-392, 1947.

[52] Joel A Paulson, Edward A Buehler, and Ali Mesbah. Arbitrary polynomial chaos for uncertainty propagation of correlated random variables in dynamic systems. IFAC-PapersOnLine, 50(1):3548-3553, 2017.

[53] Christopher M Bishop. Pattern recognition. Machine learning, 128(9), 2006.

[54] Trevor Hastie, Robert Tibshirani, and Martin Wainwright. Statistical Learning with Sparsity: The Lasso and Generalizations. Chapman \& Hall/CRC, 2015. ISBN $1498712169,9781498712163$.

[55] Bradley Efron, Trevor Hastie, lain Johnstone, Robert Tibshirani, Hemant Ishwaran, Keith Knight, Jean Michel Loubes, Pascal Massart, David Madigan, Greg Ridgeway, Saharon Rosset, J I Zhu, Robert A Stine, Berwin A Turloptiach, Sanford Weisberg, lain Johnstone, and Robert Tibshirani. Least angle regression. Ann. Stat., 32(2):407-499, 4 2004. ISSN 00905364. doi: 10.1214/009053604000000067.
[56] Evgenii Tsymbalov, Maxim Panov, and Alexander 1074 Shapeev. Dropout-based active learning for regres- 1075 sion. In International conference on analysis of images, 1076 social networks and texts, pages 247-258. Springer, 1077 2018.

[57] Nikolaus Hansen and Andreas Ostermeier. Com- 1079 pletely derandomized self-adaptation in evolution 1080 strategies. Evolutionary computation, 9(2):159-195, 1081 2001.

[58] Catherine Morris and Harold Lecar. Voltage oscilla- 1083 tions in the barnacle giant muscle fiber. Biophysical 1084 journal, 35(1):193-213, 1981.

[59] Colin Sparrow. The Lorenz equations: bifurcations, 1086 chaos, and strange attractors, volume 41. Springer Sci- 1087 ence \& Business Media, 2012.

1088

[60] Pierre Dubois, Thomas Gomez, Laurent Planckaert, 1089 and Laurent Perret. Data-driven predictions of the 1090 lorenz system. Physica D: Nonlinear Phenomena, 408: 1091 132495, 2020.

[61] Maziar Raissi, Paris Perdikaris, and George Em Karni- 1093 adakis. Multistep neural networks for data-driven dis- 1094 covery of nonlinear dynamical systems. arXiv preprint 1095 arXiv:1801.01236, 2018.

[62] Robert M May. Biological populations with nonover- 1097 lapping generations: stable points, stable cycles, and 1098 chaos. Science, 186(4164):645-647, 1974.

[63] Lars F Olsen and Anita Lunding. Chaos in the $\mathbf{1 1 0 0}$ peroxidase-oxidase oscillator. Chaos: An Interdis- 1101 ciplinary Journal of Nonlinear Science, 31(1):013119, 1102 2021.

1103

[64] Steven L Brunton and J Nathan Kutz. Data-driven sci- $\mathbf{1 1 0 4}$ ence and engineering: Machine learning, dynamical sys- 1105 tems, and control. Cambridge University Press, 2019. 1106

[65] Samay Pande, Holger Merker, Katrin Bohl, Michael 1107 Reichelt, Stefan Schuster, Luís F De Figueiredo, 1108 Christoph Kaleta, and Christian Kost. Fitness and 1109 stability of obligate cross-feeding interactions that 1110 emerge upon gene loss in bacteria. The ISME journal, 1111 8(5):953-962, 2014.

1112

[66] Neythen J Treloar, Alex JH Fedorec, Brian Ingalls, and 1113 Chris P Barnes. Deep reinforcement learning for the $\mathbf{1 1 1 4}$ control of microbial co-cultures in bioreactors. PLoS 1115 computational biology, 16(4):e1007783, 2020. 
[67] Lukas Hewing, Elena Arcari, Lukas P Fröhlich, and Melanie N Zeilinger. On simulation and trajectory prediction with gaussian process dynamics. In Learning for Dynamics and Control, pages 424-434. PMLR, 2020.

[68] Kyriakos Polymenakos, Alessandro Abate, and Stephen Roberts. Safe policy search with gaussian process models. arXiv preprint arXiv:1712.05556, 2017.

[69] Agathe Girard, Carl Edward Rasmussen, Joaquin Quinonero-Candela, and Roderick Murray-Smith. Gaussian process priors with uncertain inputs? application to multiple-step ahead time series forecasting. 2003.

[70] Anthony J Abel and Douglas S Clark. A comprehensive modeling analysis of formate-mediated microbial electrosynthesis. ChemSusChem, 14(1):344-355, 2021.

[71] Aaron J Berliner, Jacob M Hilzinger, Anthony J Abel, Matthew J McNulty, George Makrygiorgos, Nils J H Averesch, Soumyajit Sen Gupta, Alexander Benvenuti, Daniel F Caddell, Stefano Cestellos-Blanco, Anna Doloman, Skyler Friedline, Davian Ho, Wenyu Gu, Avery Hill, Paul Kusuma, Isaac Lipsky, Mia Mirkovic, Jorge Luis Meraz, Vincent Pane, Kyle B Sander, Fengzhe Shi, Jeffrey M Skerker, Alexander Styer, Kyle Valgardson, Kelly Wetmore, Sung-Geun Woo, Yongao Xiong, Kevin Yates, Cindy Zhang, Shuyang Zhen, Bruce Bugbee, Douglas S Clark, Devin ColemanDerr, Ali Mesbah, Somen Nandi, Robert M Waymouth, Peidong Yang, Craig S Criddle, Karen A McDonald, Lance C Seefeldt, Amor A Menezes, and Adam P Arkin. Towards a Biomanufactory on Mars. Frontiers in Astronomy and Space Sciences, 8:120, 2021. ISSN 2296-987X. doi: 10.3389/fspas. 2021. 711550. URL https : //www.frontiersin.org/article/ $10.3389 /$ fspas .2021 .711550 .

[72] César I Torres, Andrew Kato Marcus, Prathap Parameswaran, and Bruce E Rittmann. Kinetic experiments for evaluating the nernst- monod model for anode-respiring bacteria (arb) in a biofilm anode. Environmental science \& technology, 42(17):6593-6597, 2008.

[73] M Kazemi, D Biria, and $\mathrm{H}$ Rismani-Yazdi. Modelling bio-electrosynthesis in a reverse microbial fuel cell to produce acetate from $\mathrm{CO} 2$ and $\mathrm{H} 2 \mathrm{O}$. Phys. Chem. Chem. Phys., 17(19):12561-12574, 2015. doi: 10.1039/C5CP00904A. URL http://dx.doi.org/10. 1039/C5CP00904A.
[74] Andrew Kato Marcus, C Torres, and B Rittmann. 1164 Conduction-based modeling of the biofilm anode of 1165 a microbial fuel cell. Biotechnology and Bioengineering, 1166 98, 2007.

[75] E Borgonovo. A new uncertainty importance 1168 measure. Reliability Engineering I\& System Safety, 1169 92(6):771-784, 2007. ISSN 0951-8320. doi: 1170 https://doi.org/10.1016/j.ress.2006.04.015. URL 1171 http://www.sciencedirect.com/science/article/ 1172 pii/s0951832006000883.

[76] Marc C Kennedy and Anthony O'hagan. Bayesian cal- 1174 ibration of computer models. J. R. Statist. Soc. B, 63: 1175 425-464, 2001.

1176

[77] Jun S Liu and Rong Chen. Sequential monte carlo 1177 methods for dynamic systems. Journal of the Amer- 1178 ican Statistical Association, 93(443):1032-1044, 91179 1998. ISSN 1537274X. doi: 10.1080/01621459.1998. 1180 10473765.

[78] Richard A Davis, Keh-Shin Lii, and Dimitris N Politis. 1182 Remarks on some nonparametric estimates of a den- 1183 sity function. In Selected Works of Murray Rosenblatt, 1184 pages 95-100. Springer, 2011.

1185

[79] Diogo Rodrigues, Georgios Makrygiorgos, and Ali 1186 Mesbah. Tractable global solutions to bayesian opti- 1187 mal experiment design. In 2020 59th IEEE Conference 1188 on Decision and Control (CDC), pages 1614-1619. IEEE, 1189 2020.

[80] Joel A Paulson, Marc Martin-Casas, and Ali Mesbah. 1191 Optimal bayesian experiment design for nonlinear dy- 1192 namic systems with chance constraints. Journal of Pro- 1193 cess Control, 77:155-171, 2019.

\section{6 | SUPPLEMENTARY INFORMA- ${ }_{1195}$ TION

\section{1 | Additional Parameters for Morris-Lecar}

The extra expressions that appear in the Morris-Lecar 1199 model are given by 


$$
\begin{array}{r}
M_{\infty}=0.5\left(1+\tanh \frac{V-V_{1}}{V_{2}}\right) \\
N_{\infty}=0.5\left(1+\tanh \frac{V-V_{3}}{V_{4}}\right) \\
\lambda_{N}=\phi \cosh \frac{V-V_{3}}{2 V_{4}} .
\end{array}
$$

The Type I model parameters are given as

$\left[C_{M}, g_{L}, V_{L}, g_{C a}, V_{C a}, g_{k}, V_{k}, V_{1}, V_{2}, V_{3}, V_{4}, \phi\right]=$

$[20,2,-60,4,120,8,-84,-1.2,18,12,17.4,0.066]$

1202

\section{2 | Extra information for the MES Reactor}

Here we provide the complete mathematical description of the MES reactor. For completeness we repeat the main equations that were presented in the manuscript. The bulk quantities, with a subscript $b$, are described by:

$$
\begin{array}{r}
\frac{d L_{f}}{d t}=\left(Y \hat{q}-r_{d}\right) L_{f} \\
\frac{d S_{b}}{d t}=\frac{A_{f}}{V_{r}} j_{S} \\
\frac{d P_{b}}{d t}=\frac{A_{f}}{V_{r}} j_{P},
\end{array}
$$

where the various variables are described in the text. The mass balances for the substrate and product are a function of the flux of each species across the linear boundary layer, given by

$$
j_{m}=\frac{D_{b}}{L_{b}}\left(m_{f}\left(z=L_{f}\right)-m_{b}\right), \quad m=S, P,
$$

$\mu=\mu_{\max }\left(\frac{1}{1+\exp \left(\frac{-F \eta_{f}}{R T}\right)}\right)\left(\frac{S_{f}}{k_{s}+S_{f}\left(1+\frac{S_{f}}{k_{i}}\right)}\right) \max \left(0,1-\frac{P_{f}}{P_{\max }}\right)$

where $k_{s}$ is the affinity/Monod constant, $k_{i}$ is a sub- 1215 strate inhibition constant and $P_{\max }$ is a maximum prod- 1216 uct concentration. $F$ is the Faraday constant. Therefore, 1217 we consider inhibition both by the substrate and the 1218 product. Under the pseudo-steady state assumptions, 1219 the following differential equations are solved within 1220 the biofilm:

\section{- Substrate Concentration}

We have the differential conservation law, the boundary condition at the biofilm edge as well as a no flux condition at the cathode given by

$$
\begin{array}{r}
D_{f} \frac{d^{2} S_{f}}{d z^{2}}-X \mu-\frac{1}{Y_{P / S}}(\alpha \mu+\beta) X=0 \\
D_{f} \frac{d S_{f}}{d z}\left(z=L_{f}\right)=\frac{D_{b}}{L_{b}}\left(S_{f}\left(z=L_{b}\right)-S_{b}\right) \\
D_{f, S} \frac{d S_{f}}{d z}(z=0)=0,
\end{array}
$$

respectively

\section{- Product Concentration}

Similar to the substrate, we have the following equations

$$
\begin{array}{r}
D_{f} \frac{d^{2} P_{f}}{d z^{2}}+(\alpha \mu+\beta) X=0 \\
D_{f} \frac{d P_{f}}{d z}\left(z=L_{f}\right)=\frac{D_{b}}{L_{b}}\left(P_{f}\left(z=L_{b}\right)-P_{b}\right) \\
D_{f} \frac{d P_{f}}{d z}(z=0)=0,
\end{array}
$$

Note that it has been assumed that the diffusivity 1225 of substrate and product within the film is equal, de- 1226 noted by $D_{f}$.

- Overpotential

Finally, we have the equations for the overpotential; We describe the diffusion of electrons in the conductive biofilm, we have a fixed voltage at the cathode 
and no-flux at the biofilm edge

$$
\begin{array}{r}
k_{\text {bio }} \frac{d^{2} \eta_{f}}{d z^{2}}-\frac{F \gamma_{1}}{t_{c o n v}} f_{e, 0} X \mu+\frac{F \gamma_{2}}{t_{c o n v}} X r_{r e s}=0 \\
\frac{d \eta_{f}}{d z}\left(z=L_{f}\right)=0 \\
\eta_{f}(z=0)=\eta_{0},
\end{array}
$$

1228 respectively. 